\title{
Bestimmung der Sonnenparallaxe aus dem Verhältniss der Erd- zur Sonnenmasse.
}

Die Massenwirkung der Erde, wie die der Sonne ist in Bezug auf eine besondere Längen-Einheit jede für sich mit grosser Schärfe zu bestimmen, jene durch die experimental bestimmte Länge des einfachen Secundenpendels auf der ihrer Gestalt und Grösse nach durch Gradmessungen bekannten Erde, letztere, für die mittlere Entfernung der Sonne von der Erde als Mass-Einheit aus der Umlaufszeit - der Länge des siderischen Jahres. Das Verhältniss dieser beiden Massenwirkungen hängt dann nur von dem Verhältniss des Erdhalbmessers zur Entfernung der Erde von der Sonne, d. i. von der Parallaxe ab.

Andererseits wird das Verbältniss der Erd- zur Sonnenmasse durch die aus Beobachtungen direct zu ermittelnden Störungen bekannt und es hängt nur von der Schärfe der relativen Bestinumungen $a b$, ob die Störungen mittelst der Parallaxe oder diese aus jenen zu hestimmen sind. Auffallende Abweichungen müssen zu weiteren Forschungen veranlassen.

$Z$ u verschiedenen Zeiten ist so die Sonnenparallaxe aus den Mondstörungen (parallactische Ungleichbeiten) bestimmt worden. Bei den Störungen der Planeten durch die Erde muss die vereinigte Wirkung der Erd- und Mondmasse in Betracht gezogen werden; da letztere jedoch nur einen geringen und verhältnissmässig sicher zu bestimmenden Theil der ersteren ausmacht, so ist auch die hierdurch entstehende Ungenauigkeit von verschwindend kleinem Einfluss. Bei denjenigen Störungen, die wir als Säcularänderungen der Bahnelemente betrachten können, wirken in verschiedener, von einander unabhängiger Weise alle Planetenmassen ein, so dass die zu ermittelnde Wirkung der Erdmasse von der Genauigkeit unserer Kenntniss der anderen mit abbängig ist.

Die in den Annales de l'Observatoire impérial de Paris, Tome VI. gegebenen Elemente und Tafeln der Venusbahn sind aus Meridian-Beobachtungen in Greenwich und Paris, 1836-1858, für 1850 abgeleitet und ihre Veränderungen aus den Planetenmassen:

$$
\begin{aligned}
& \not \ldots . . m=0,0000003333(1+v) \\
& \text { ㅇ….m } m^{r}=0,0000024885\left(1+v^{x}\right) \\
& \text { t.....m } m^{I I}=0,0000028174\left(1+v^{I I}\right) \\
& \sigma^{x} \ldots m^{I I I}=0,0000003319\left(1+v^{I I I}\right) \\
& 4 \ldots m^{I V}=0,000952381 \quad\left(1+v^{x V}\right) \\
& \text { Ђ....m } m^{v}=0,000284738 \cdot\left(1+v^{v}\right)
\end{aligned}
$$

wo $(1+v),\left(1+v^{r}\right)$ u. s.w. Verbesserungscoefficienten bedeuten, deren nähere Bestimmung vorbehalten blieb. In den Säcularänderungen der Tafeln sind die Werthe $v, v^{t}$ u. s. w. gleich 0 angenommen.
Bei der Vergleichung der hiernach berechneten Zeiten der Ein- und Austritte in den Durchgängen der Venus durch die Sonne von 1761 und 1769 (Astr. Nachr. Bd. 76, p. 264) zeigten sich aufällige Abweichungen, welche nur durch Aenderung der berechneten Knotenlänge zu heben waren. Die aus den Durchgängen folgende Knotenlänge ist selbst mit Rücksicht auf eine Unsicherheit der Sonnenparallaxe von 0"1 auf $2^{\prime \prime}$ sicher, während der Unterschied derselben von der berechneten 34 " heträgt.

Obwohl die Bestimmung dieses Elenentes aus Meridianbeobachtungen der Venus wegen mangelhafter Beleuchtung des Nord- und Südrandes in grösserer Erdnähe des Planeten unsicherer wird, so scheint doch die Grösse des abweichenden Werthes aus den Durchgängen durch die Ungenauigkeit der ersteren nicht erklärbar. Isne Meridian-Beobachtungen ergeben zwar, die Greenwicher wie die Pariser, nach den Mittheilungen derselben (Ann. Tome VI., pag. 51-55), für den aufsteigenden Knoten einen um etwa $18^{\prime \prime}$ grösseren Werth als für den niedersteigenden, doch dieser Unterschied wird wohl erklärlich aus dem bei der Reduction der Beobachtungen angewandten zu kleinen Werthe der Parallaxe (die aus dem damals noch allgemein angewandten Werthe der Sonnenparallaxe $8^{\prime \prime 57}$ folgte). Im Mittel aus allen Beobachtungen wird bei der grösseren und im $\Omega$ und $\vartheta$ nahe gleichen Zahl derselben, der Werth der Knotenlänge in engeren Grenzen sicher sein. Ebenso würden sich Ungenauigkeiten in der Annahme des Durchmessers der Venusscheibe bei gleicher Zahl der Beobachtungen vor und nach dem Knotendurchgange behen. Für eine genaue Bestimmung der Knotenlänge aus Meridian-Beobachtungen müssten diese Umstände in sorgfältige Betrachtung gezogen werden. Hier genüge vorläufig der Nachweis, dass ein Fehler von nahe $34^{\prime \prime}$ in der für 1850 ermittelten Knotenlänge nicht gefunden werden kann; er muss daher in der Veränderung der Knoteulänge von 1761 bis 1850 (88,56 Jahre) gesucht werden.

Für die jährliche Veränderung der Knotenlänge giebt Leverrier (Ann. Tome VI., pag. 23) folgenden Werth:

$$
\begin{array}{r}
+32^{\prime \prime} 8899+0^{\prime \prime} 1116 v-5^{\prime \prime} 0369 v^{I}-6^{\prime \prime} 7199 v^{I Z}-0^{\prime \prime} 1975 v^{I X I} \\
-5^{\prime \prime} 2245 v^{I V}-0^{\prime \prime} 2736 v^{\nabla}-0^{\prime \prime} 0042 v^{\nabla I}-0^{\prime \prime} 0010 v^{\text {rII }}
\end{array}
$$

Bei der geringen Grösse der Coefficienten von $v$ und $v^{\text {IIr }}$ kann in den Massenanuahmen für Mercur und Mars keine Aenderung angenommen werden, durch welche die Zahl 32"8899 sich um 0"4 $\left(=\frac{34 "}{88,56}\right)$ verkleinerte. Der Fehler der Marsmasse, hier bereits nach (Ann. Tome VI., pag. 96) verbessert, beträgt sicher weniger als $\frac{1}{10}$ ihres Werthes; nach Encke (Jahrbuch 1861 , pag. 337 und 342 ) würde $v=-0.1$ 
zu setzen sein. Die Massen von Jupiter und $S$ aturn sind so genau hestimmt, dass zulässige Werthe von $v^{t \tau}$ und $v^{t}$ noch weniger etwas bewirken.

Die obige Venus masse hat Leverrier in naher Uebereinstimmung mit Barkhardt aus den Erdstörungen angenommen und aus seinen Vergleichungen bei der Erdbahn (Ann.T.IV., pag. 96) eine nur geringe Vergrösserung $\left(v^{x}=+0,004\right)$ gefunden. Das Maximum der Störungen kurzer Periode der Erde durch Venus beträgt etwa 12", so dass eine genaue Bestinumung von $v^{x}$ auf 0,005 durch zahlreiche gute Beobachtungen wobl mäglich sein wird. Aus den Säcularänderungen der Schiefe seit Bradley folgt ein kleinerer Werth der Venusmasse. In den Sonnentafeln von Hansen und Olufsen ist ein Werth angenommen, aus welchen sich $v^{x}=-0,015$ ergeben würde. Mit diesem Werthe werden auch die Bestimmungen der Schiefe seit Bradley bis auf die neueste Zeit zu befriedigender Uebereinstimmung gebracht; eine Vergleichung der Dorpater Beobachtungen von $W$. Struve 1823 his 1827 ergab mir eine gute Bestätigung des letzteren Werthes. *) Eine sicherere Bestimmung der Venusmasse ist zur Bestimmung der Sonnenparallaxe aus den Säcularänderungen von wesentlichster Bedeutung.

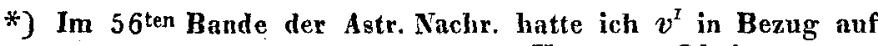
die Annahme in den Sonnentafeln Hansen-Olufsen gleich $+0,05$ angegeben; dieser Werth ist jedoch noch mit dem Maximum der Störungswerthe, nach der vorhergegangenen Annahme mit 14,3 zu dividiren.
Nach den Daten, welche Encke in den Abhandlungen der Berliner Akademie für 1842 giebt, wird die Masse der Erde, ohne Mondsmasse, $m^{\circ}=\{1,64512-10\} p_{0}^{3}$, wo der Logarithmus $\{\ldots\}$ als Constante betrachtet werden kann und $\boldsymbol{p}_{0}$ den Werth der mittleren Horizontalparallaxe der Sonne bezeichnet. Mit $p_{0}=8 " 57116$ erhält man hieraus $m^{0}=0,00000278125$ und setat man $\mu$, die Masse des Mondes, gleich $\frac{1}{80}$ der Erdmasse, so wird $m^{I I}=m^{0}+\mu=$ 0,0000028160 . Die Abweichung dieses Werthes von den in den Venustafeln angenommenen ist so gering, dass sie in der Knotenlänge für 120 Jahre nur $0 " 1$ ausmacht.

Wir erhalten nun, unter der Voraussetzung, dass die angenommenen Massen ausser der der Erde, wie die Knotenlänge aus den Tafeln für 1851 richtig seien, aus dem Unterschied letzterer mit der Knotenlänge für den Durchgang 1761 und der Zwischenzeit: 88,56 Jabren:

$$
\begin{gathered}
6^{\prime \prime} 7199 v^{I I}=\frac{34^{\prime \prime} 5}{88,56}=0,389 j \\
1+v^{I I}=\frac{p_{0}^{\prime 3}}{p_{0}^{3}}=1+3 \frac{\Delta p_{0}}{\frac{I}{2}\left(p_{0}+p_{0}^{\prime}\right)} ; p_{0}=8,57116
\end{gathered}
$$

woraus

$$
p_{0}^{\prime}=8^{\prime \prime} 74
$$

folgt. Eine Aenderung von $+1^{\prime \prime}$ in der Knotenlänge für 1850 giebt eine Aenderung des Werthes von $p_{0}: \Delta p_{0}=+0,0047$. Mit der Aunahme von $v^{x}=-0,015$ wärde man $p_{0}^{\prime}=8^{\prime \prime} 77$ erhalten.

C. Powalky.

\section{Verbesserte Bahn der Janthe (98).}

Da die bevorstehende Opposition der Janthe für die Beobachtung eine sehr vortheilhafte sein wird, wegen der grossen Annäberung und folglich Lichtstärke des Planeten, so schien es zweckmässig, das sämmtliche bis jetzt vorhandene Material zu einer verbesserten Bahn zusammen zu fassen. Der Planet wurde entdeckt am 18. April 1868, und beobachtet bis zum 18. Juni. Aus dieser ersten Erscheinung liegen vor: $11 \mathrm{Beoh}-$ achtungen von Hamilton College (Astr. Nachr. № 1755), 2 Beobachtungen von Washington (Astr. Nachr. No 1707, und gleichlautend in den Washington Observations), und 5 von Berlin (Astr. Nachr. No 1764). Eine in den Astr. Nachr. No 1722 aus Leipzig mitgetheilte Beobachtung stellt sich heraus als wohl nicht zu den Planeten gehörig; sie wird a. a. 0 . auch schon vom Beobachter selhst als in dieser Hinsicht fraglich bezeichnet. In der zweiten Opposition, im Jahre 1869, hatte Jan the die Declination von $-42^{\circ}$, ging also unbeobachtet vorüber, da keine der Sternwarten auf der südlichen Hemisphäre sich mit den kleinen Planeten zu beschäftigen scheint. Zur Zeit der dritten Opposition, 1870, wurde der Ort des Planeten bier in Hamilton College 5 Mal bestimmt (Astr. Nachr. No 1826); andere Beobachtungen sind mir nicht zu Gesicht gekommen.

Die Sternörter sind theilweise corrigirt worden, zufolge der Daten, die mir jetzt die besten zu sein scheinen. Dabei ward indess auf die sogenannten, systematischen Correctionen" keine Rücksicht genommen.

Zur Bildung von Normalorten wurden die folgenden (schon in den Astr. Nachr. M\& 1708 publicirten) Elemente:

Epoche: 1870 Januar 0,0 Berliner M. Zt.

$$
\begin{aligned}
M_{\mathrm{o}} & =166^{\circ} 30^{\prime} 14^{\prime \prime} 68 \\
\pi & =1474448,08 \\
\beta & =3541823,66 \\
i & =153236,10 \\
\varphi & =105419,82 \\
\mu & =806^{\prime \prime} 683 \\
\log a & =0,4288691
\end{aligned} \text { M. Aeq. } 1870,0
$$

zu Grunde gelegt, damit die Störungen durch Jupiter berechnet, und die Beobachtungen strenge verglichen, wie im Tableau zusammen gestellt ist. 